\title{
Anti-microbial Activity of Urine after Ingestion of Cranberry: A Pilot Study
}

\author{
Yee Lean Lee ${ }^{1}$, Wadie I. Najm², John Owens ${ }^{1}$, Laurie Thrupp ${ }^{1}$, Sheryl Baron ${ }^{1}$, \\ Edward Shanbrom ${ }^{1}$ and Thomas Cesario ${ }^{1}$
}

${ }^{1}$ Department of Medicine, Division of Infectious Disease and ${ }^{2}$ Department of Family Medicine \& Geriatrics, Irvine School of Medicine, University of California, CA, USA

We explore the anti-microbial activity of urine specimens after the ingestion of a commercial cranberry preparation. Twenty subjects without urinary infection, off antibiotics and all supplements or vitamins were recruited. The study was conducted in two phases: in phase 1, subjects collected the first morning urine prior to ingesting $900 \mathrm{mg}$ of cranberry and then at 2, 4 and $6 \mathrm{~h}$. In phase 2, subjects collected urine on 2 consecutive days: on Day 1 no cranberry was ingested (control specimens), on Day 2, cranberry was ingested. The $\mathrm{pH}$ of all urine specimens were adjusted to the same $\mathrm{pH}$ as that of the first morning urine specimen. Aliquots of each specimen were independently inoculated with Escherichia coli, Klebsiella pneumoniae or Candida albicans. After incubation, colony forming units $/ \mathrm{ml}\left(\mathrm{CFU} \mathrm{ml} \mathrm{m}^{-1}\right)$ in the control specimen was compared with $\mathrm{CFU} \mathrm{m} \mathrm{l}^{-1}$ in specimens collected 2, 4 and $6 \mathrm{~h}$ later. Specimens showing $\geq 50 \%$ reduction in CFU ml ${ }^{-1}$ were considered as having 'activity' against the strains tested. In phase 1, 7/20 (35\%) subjects had anti-microbial activity against E. coli, 13/20 (65\%) against $K$. pneumoniae and 9/20 (45\%) against $C$. albicans in specimens collected 2-6h after ingestion of cranberry. In phase 2, 6/9 (67\%) of the subjects had activity against $K$. pneumoniae. This pilot study demonstrates weak anti-microbial activity in urine specimens after ingestion of a single dose of commercial cranberry. Anti-microbial activity was noted only against $K$. pneumoniae $2-6 \mathrm{~h}$ after ingestion of the cranberry preparation.

Keywords: anti-microbial activity-cranberry-urinary tract infection

\section{Background}

Cranberry (Vaccinium macrocarpon) fruits and leaves have a long history of traditional use in folk medicine for the management of diverse conditions including urinary problems, wounds, stomach problems, diabetes, etc. Over the last decades popular interest in the use of cranberry for the prevention and treatment of urinary tract infections (UTI) has been on the rise. Sales of cranberry in 2005 exceeded $\$ 15$ million and cranberry was ranked the fifth highest selling herb in the US (1).

For reprints and all correspondence: Wadie Najm, MD, MSEd, 200 S. Manchester \#512, Orange, CA 92868, USA. Tel: +714-456-5171;

Fax: +714-456-7984; E-mail: winajm@uci.edu
Scientific interest in cranberry followed popular interest closely. A PubMed search over the last 36 years (1970-2006), limited to title, identified 185 papers on cranberry, of which, 22 were randomized controlled trials, and two Cochrane reviews. The majority of published controlled trials explore the effectiveness of cranberry for the prevention of UTI (2). Cochrane reviewers concluded that evidence from two well-designed studies indicates that cranberry juice decreased the number of symptomatic UTIs over a 12-month period. Few studies looked at the use of cranberry for the treatment of UTI, however well-designed studies are yet to be conducted as indicated by the 2004 Cochrane review (3). No major adverse effects or interactions were reported or identified in these reviews or more recent studies $(4,5)$. 
Table 1. Anti-bacterial activity of cranberry PVP versus PVP in Broth

\begin{tabular}{|c|c|c|c|c|c|c|}
\hline \multirow[t]{2}{*}{ Strains } & \multirow{2}{*}{$\begin{array}{l}\text { CB PVP } \\
\text { Batch No. }\end{array}$} & \multirow{2}{*}{$\begin{array}{l}\text { Broth only } \\
\text { CFU ml }\end{array}$} & \multicolumn{2}{|c|}{ PVP broth } & \multicolumn{2}{|c|}{ Cranberry PVP broth } \\
\hline & & & Dilution & $\overline{\mathrm{CFU} \mathrm{ml}} \mathrm{m}^{-1}$ & Dilution & $\mathrm{CFU} \mathrm{ml}^{-1}$ \\
\hline \multirow[t]{2}{*}{ E. coli (ATTC 295220) } & 1 & $2 \times 10^{9}$ & $1: 2$ & $1.7 \times 10^{8}$ & $1: 2$ & $1 \times 10^{7}$ \\
\hline & 3 & $1.9 \times 10^{9}$ & $1: 2$ & $1.8 \times 10^{8}$ & $1: 2$ & $1 \times 10^{7}$ \\
\hline K. pneumonia (ATCC 13883) & 4 & $4.3 \times 10^{8}$ & $1: 2$ & $8.5 \times 10^{7}$ & $1: 2$ & $<10^{2}$ \\
\hline S. aureus (ATCC 29213) & 4 & $1 \times 10^{8}$ & $1: 2$ & $1.8 \times 10^{8}$ & $1: 2$ & $1.6 \times 10^{1}$ \\
\hline
\end{tabular}

Initial inoculate approximately $10^{5} \mathrm{CFU} \mathrm{ml}^{-1}$. Overnight incubation at $35^{\circ} \mathrm{C}$.

With mounting interest in the clinical use of cranberry, scientific curiosity about cranberry's anti-microbial mechanism of action grew. The exact anti-microbial action of cranberry remains under investigation. Initial proposed mechanism attributed the anti-microbial benefits to hippuric acid which has the potential to acidify the urine (6). Later studies confirmed that cranberry juice can lower urinary PH $(7,8)$. More recently, studies suggested that cranberry's activity is related to the inhibition of bacterial adherence to the uroepithelium. Recent evidence suggests that fructose, found in cranberries, interferes with adhesion of type 1 fimbriated (mannose-sensitive) Escherichia coli to the uroepithelium (9), while other studies show that proanthocyanidins in cranberries inhibit the adherence of p-fimbriated (mannose-resistant) E. coli to the uroepithelium $(10,11)$. In fact, recent studies suggest that cranberry juice irreversibly inhibits p-fimbriae preventing attachment of $E$. coli to the uroepithelium (12).

In addition to the anti-adhesive effect, a possible antimicrobial activity has been suggested. In effect a laboratory study verified that concentrated cranberry juice had a direct anti-microbial activity in vitro (13). However, despite the encouraging outcomes reported in the prevention studies and in laboratory studies looking at mechanism of action, evidence of direct anti-microbial activity in the urine after ingestion of cranberry has yet to be demonstrated.

To evaluate the anti-microbial effect of urine specimens after ingestion of cranberry we conducted a pilot study assaying the direct anti-microbial activity of urine specimens taken from volunteer subjects after ingestion of a commercially available cranberry product.

\section{Methods}

This study proposes to evaluate the in vitro bioactivity (anti-microbial) of urine samples after ingestion of cranberry. The protocols were approved by the institutional review board. Twenty asymptomatic adults were recruited for the entire study, all of them completed phase 1 , and nine completed phase 2 . The study team was blinded to the identity of the subjects. All subjects were asked to abstain from taking any cranberry, health food fruit extracts, anti-oxidants, vitamins or minerals and antibiotics for 1 and 2 weeks, respectively, prior to particpating in the studies and during specimen collection. Subjects were also asked to complete a food diary starting 3 days prior and on the day(s) of specimen collections.

\section{Product Used}

A commercially available cranberry softgel 'TruNature' was used in this study. Each $300 \mathrm{mg}$ softgel contained of a mixture of dry whole cranberry extract $(275 \mathrm{mg})$ and ShanStar Concentrate cranberry extract $(25 \mathrm{mg})$. ShanStar Concentrate extract is a highly purified preparation of cranberry proanthocyanins on crosslinked polyvinylpyrrolidone (PVP) substrate. During the preparation, PVP is mixed into normal cranberry juice. The proanthocyanins and other polyphenolic compounds have a very high affinity for the PVP molecule. The PVPcranberry (CB-PVP) is then removed from the juice by filtration and dried. HPLC analysis of ShanStar Concentrate extract shows several 100 -fold increase in the concentration of the proanthocyanins and other polyphenolic compounds when compared to normal juice without the high level of sugars and organic acids usually found in cranberry juice (14). To ensure that the activity seen in the test is from cranberry and not from PVP, we conducted a small comparison of the antibacterial activity of PVP versus cranberry PVP (Table 1). The results confirm that the anti-bacterial activity seen is from cranberry.

\section{Study Conducted}

The study was conducted in two phases. In the phase 1, urine was collected for 1 day only. On the day of the study, the first morning urine was collected as a baseline contol urine. Subjects were then asked to take a commercial cranberry product (TruNature cranberry $300 \mathrm{mg}$ softgels). Each subject was asked to take three 


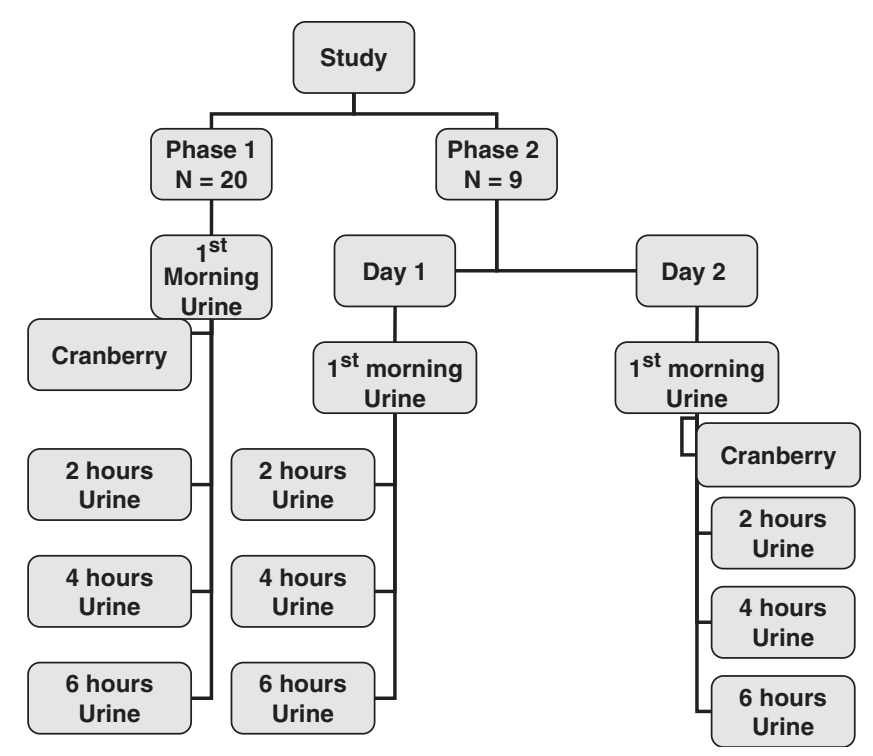

Figure 1. Time line for urine collection.

softgels $(900 \mathrm{mg})$ once in the morning. Additional urine samples were then collected at $2 \mathrm{~h}$ inetrvals over $6 \mathrm{~h}$, for a total of three samples (at 2, 4 and $6 \mathrm{~h}$ post-ingestion of cranberry) (Fig. 1). Each subject was asked to immediately refrigerate all samples after testing and recording each specimens' specific gravity and $\mathrm{PH}$. All specimes and data were confidentially coded. Thirteen subjects were enrolled in this phase of the study.

In phase 2, the protocol was amended and subjects were asked to collect urine specimens for two subsequent days. On Day 1, urine was collected at $2 \mathrm{~h}$ intervals, as in phase 1, but without cranberry ingestion (control specimens). On Day 2, the protocol remained the same as that of phase 1, (i.e. urine was collected at $2 \mathrm{~h}$ intervals, as in phase 1, but with cranberry ingestion, see Fig. 1). Thus, in this phase of the study we were able to compare the anti-microbial activity in urine samples collected on 2 subsequent days with and without ingestion of cranberry. Nine subjects were tested; two subjects were already enrolled in phase 1 of the study and consented to repeat the sample collections for phase 2 .

\section{Microbiological Assays}

In the laboratory, urines were filter-sterilized, aliquots of each of the specimens was then assayed for anti-microbial activity in vitro against a variety of common bacterial organisms which can be the cause of human UTI.

The $\mathrm{pH}$ of all urine specimens was adjusted to the same $\mathrm{pH}$ as the baseline control specimens and filtered. Aliquots of each specimen were independently inoculated with $10^{2}-10^{3}$ cell $\mathrm{ml}^{-1}$ each of single strains of E. coli, Klebsiella pneumoniae and Candida albicans. Bacterial strains tested included E. coli ATCC 29522,
$K$. pneumoniae ATCC 13883 and clinical strain $C$. albicans from University of California at Irvine medical center. Overnight cultures were incubated at $35^{\circ} \mathrm{C}$ on tryticase soy agar media were used as inocula.

A low inoculum of bacteria was used deliberately to mimic colonization and not an established infection. The organisms were the same strains utilized in our pilot study (13). After $24 \mathrm{~h}$ of incubation for E. coli and $K$. pneumoniae, and $48 \mathrm{~h}$ for C. albicans, colony forming units per milliliter $\left(\mathrm{CFU} \mathrm{ml} \mathrm{m}^{-1}\right.$ ) of each specimen were enumerated by subculture with quantitative plate counts in duplicate. The number of $\mathrm{CFU} \mathrm{ml} \mathrm{m}^{-1}$ in the baseline control specimens (early morning urine specimen) were compared with CFU ml ${ }^{-1}$ in specimens collected 2, 4 and $6 \mathrm{~h}$ after ingestion of cranberry. Specimens that showed $\geq 50 \%$ reduction in CFU ml ${ }^{-1}$ compared with its controls were considered as having 'activity' against the strains tested.

\section{Data Analysis}

Data was analyzed in two parts: in part one data from all 20 subjects were included comparing the anti-microbial activity of samples 2, 3 and 4 (all after ingestion of cranberry) to the morning urine sample (control specimen taken before ingestion of cranberry). In part two, nine subjects who collected samples as per phase 2 protocol (2-day urine collection) were included. Morning urine specimens were used as the controls in each of the 2 days of this phase. Anti-microbial activity at 2, 4 and $6 \mathrm{~h}$ were compared with the morning specimen (as control) of their respective day. Presence of anti-microbial activity was defined as $50 \%$ reduction in $\mathrm{CFU} \mathrm{ml} \mathrm{m}^{-1}$ between the first morning urine specimen (control) and subsequent specimens collected at 2,4 and $6 \mathrm{~h}$. The statistic method used was $\mathrm{McNemar}$ chi-squared test.

\section{Results}

\section{Phase 1}

Data from all 20 subjects were initially analyzed comparing morning urine anti-microbial activity to that seen after ingestion of cranberry. Four men and 16 women age range 36-72 years, provided urine specimens. Anti-microbial activity ( $\geq 50 \%$ reduction in CFU from control specimen) against $E$. coli strain was identified in 7 of the $20(35 \%)$ subjects studied. Anti-microbial activity was also seen in $13 / 20$ subjects $(65 \%)$ for $K$. pneumoniae strain and in $9 / 20(45 \%)$ for C. albicans. Timing of the observed activity varied and was seen at 2, 4 or $6 \mathrm{~h}$ : however, most activities were noted $2 \mathrm{~h}$ after ingestion of cranberry (Fig. 2). The activity was generally modest but ranged from $<1 \log _{10}(50 \%)$ reduction to $2 \log _{10}(99 \%)$ reduction in $\mathrm{CFU}$. 


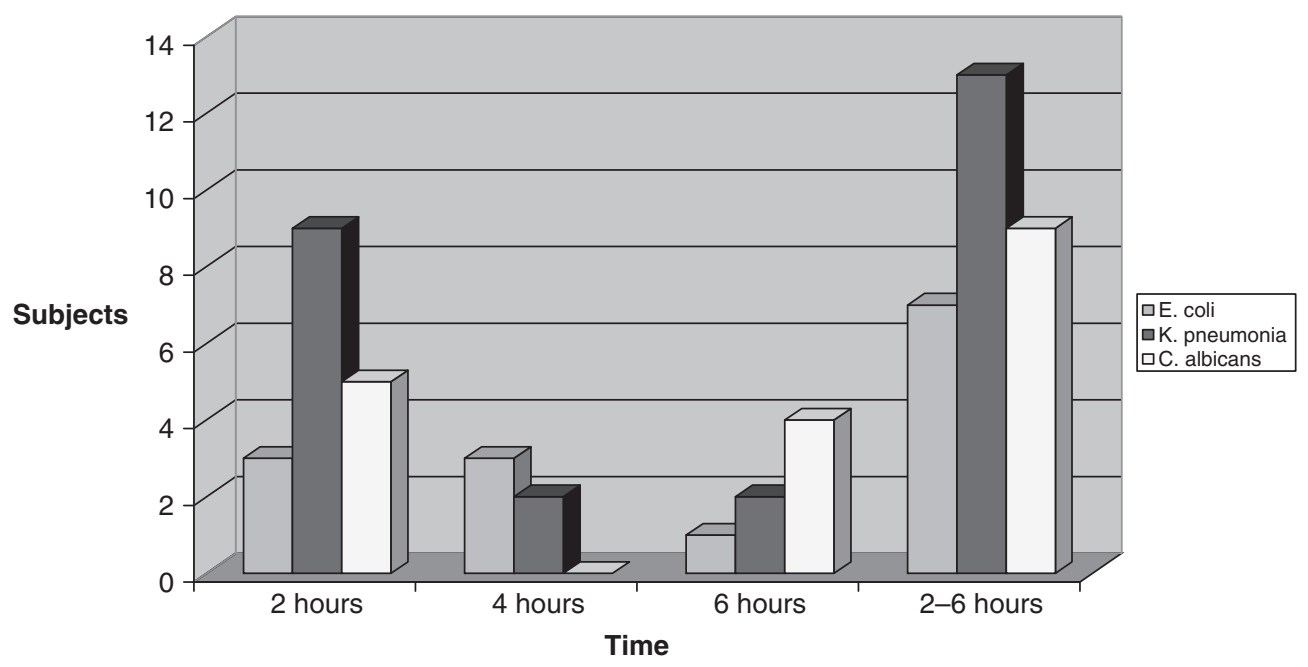

Figure 2. Anti-microbial activity found in urine after ingestion of cranberry compared with first morning urine (phase 1). Only maximal antimicrobial activity identified for each participant is reported.

\section{Phase 2}

In the second phase of the study, nine subjects provided urine specimens, seven women and two men. Analysis of specimens provided during the second phase of the study showed no significant difference in anti-microbial activity between control (Day 1 without cranberry) and test (Day 2 with cranberry) urine specimens for E. coli and C. albicans. Anti-microbial activity against K. pneumoniae was noted in six out of nine subjects $(67 \%)$ on day 2 after cranberry ingestion compared to $2 / 9(22 \%)$ on day 1 (no cranberry) (Fig. 3). Due to the small sample size, the difference was not statistically significant.

No difference in activity between men and women was noted, although the number of men sampled was too small for adequate comparison. Selection of study volunteers was not stratified by age group. None of the enrolled subjects withdrew prior to completing the study. No side effects were reported throughout the study.

\section{Discussion}

Cranberry is commonly recommended for treatment/ prevention of UTIs. Cochrane reviews of clinical studies concluded that evidence supports a role for cranberry in the prevention of UTIs, but not in the treatment of an established infection $(3,15)$. Proposed mechanisms of action included anti-adherance (9-12,16,17), antibacterial (13), changes in urine acidity $(\mathrm{PH})(7,8)$ and activation of the nitric oxide pathway. Laboratory studies identified a direct anti-bacterial activity of cranberry (13), however little is known whether this activity is maintained after ingestion. In this study, we explored the presence of anti-microbial activity in the urine of subjects after ingestion of cranberry.
For this study, we used an inoculation of $10^{2}-10^{3}$ instead of the standard $10^{5}$. This decision was reached after careful review of the published data and the Cochrane reviews which concluded that cranberry was more effective in the prevention (eliminate colonization) rather than treatment of UTI.

We postulated that the anti-bacterial effect is due to the anthocyanins and proanthocyanidins fractions of cranberry. Urine samples were collected at 2, 4 and $6 \mathrm{~h}$ after ingestion of cranberry. A recent study by Ohnishi et al. (18) demonstrated the urinary levels of anthocyanins reached a maximum between 3 and $6 \mathrm{~h}$ after ingestion of cranberry.

Our study suggests that anti-bacterial activity against $E$. coli after ingestion of cranberry was not significant. This confirms findings from two recent published studies exploring the anti-bacterial activity of urine after ingestion of cranberry $(14,19)$. Both studies adjusted the specimens for urine $\mathrm{PH}$. In the study by Monroy-Torres et al. (14), 20 young (19-24 years old) healthy women provided urine samples before and $3 \mathrm{~h}$ after taking $250 \mathrm{ml}$ of cranberry juice. Urine samples were incubated for $1-2 \mathrm{~h}$ only then plated. No significant drop in the number of $E$. coli colonies were seen. In the study by Tong et al., 10 young healthy Chinese adults (mean age $20.4 \pm 1.2$ years) collected urine at $3 \mathrm{~h}$ intervals on 2 consecutive days. On day 2 subjects drank $750 \mathrm{ml}$ of cranberry juice. Urine samples were inoculated with $2.8 \times 10^{7}$ E. coli and incubated for $24 \mathrm{~h}$. No statistically significant differences were observed between the control (day 1) and urine specimens collected on day 2 after ingestion of the cranberry juice (19). Even though the studies used different forms (juice and tablets) and doses of cranberry, the final result was the same regardless of the time of urine collection after cranberry ingestion. 


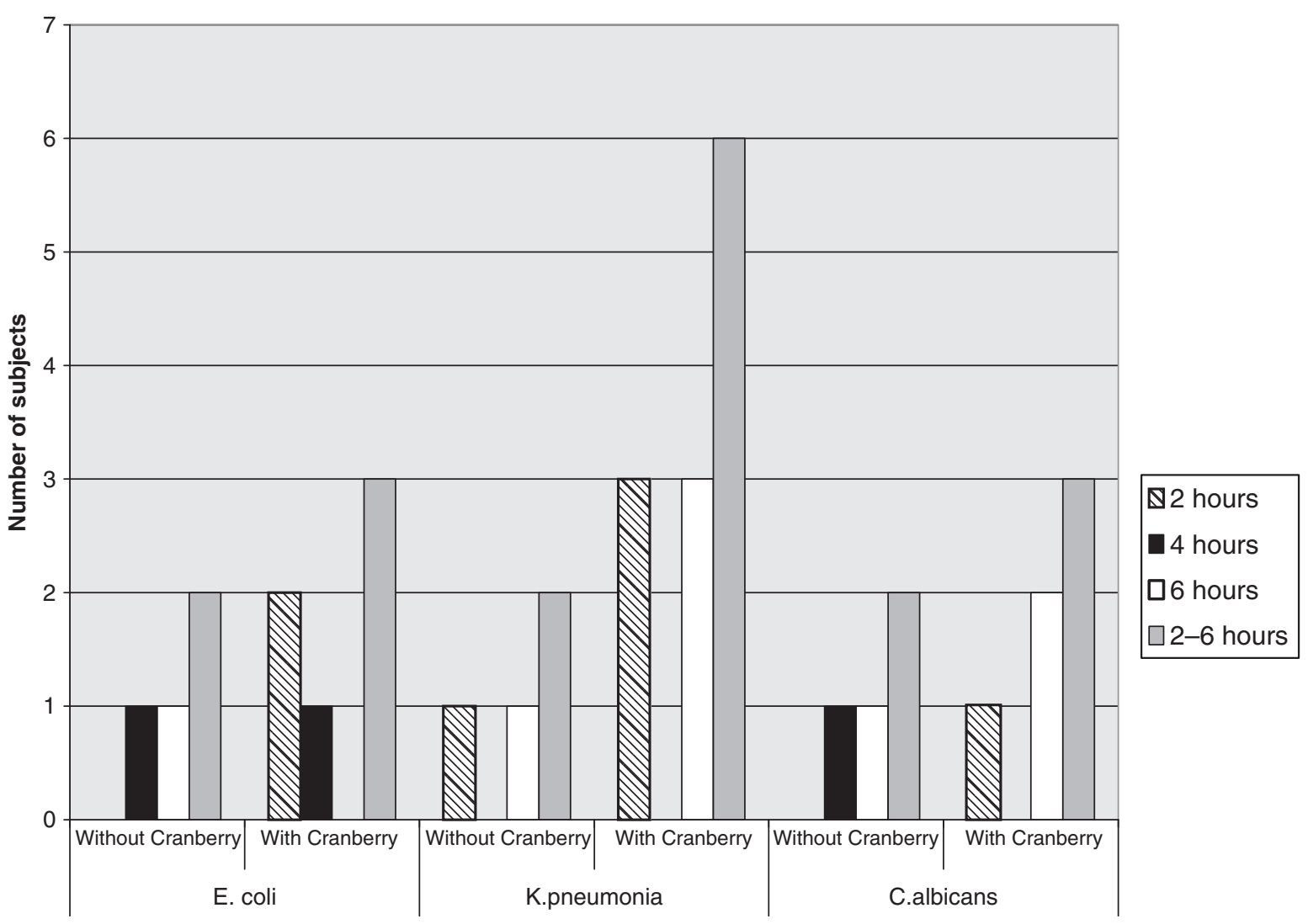

Figure 3. Anti-microbial activity found in urine with and without ingestion of cranberry on 2 subsequent days (phase 2). Only maximal antimicrobial activity identified per participant each day is reported.

In this study we also explored the presence of antimicrobial activity against less common causes of UTI, such as $C$. albicans and $K$. pneumonia. No significant drop in the number of colonies was observed for Candida after ingestion of cranberry. However, anti-microbial activity against $\mathrm{K}$. pneumonia was detected in the urine of six out of nine subjects after ingestion of the cranberry capsules. It is of interest that the occurrence of antimicrobial activity against $K$. pneumonia was noted when samples were compared in phase 1 of the study (using first morning urine specimen as control), and phase 2 (comparing samples from both days to their respective morning control specimen). Timing of the observed activity in all three strains tested varied among the 2, 4 or $6 \mathrm{~h}$ specimens; however, activity was mainly observed in the urine specimens collected $2 \mathrm{~h}$ after ingestion of the capsules. Based on this result one can postulate that the anti-microbial activity found in this study against $K$. pneumoniae is probably due to factors other than the anti-adherence activity reported for $E$. coli.

During phase $1,35 \%, 45 \%$ and $65 \%$ of specimens collected form the 20 study subjects showed anti-microbial activity in the urine against E. coli, C. albicans and $K$. pneumoniae respectively. This activity occurred $2-6 \mathrm{~h}$ after ingestion of cranberry capsules. In the second phase, $23 \%, 33 \%$ and $67 \%$ of the specimens showed some anti-microbial activity against E. coli, C. albicans and $K$. pneumoniae respectively. It is of interest that the most frequent occurrence of anti-microbial activity occurred in both phases against $K$. pneumoniae. Thus, in the CB-PVP there might be a different anti-microbial factor inhibiting $K$. pneumoniae other than the anti-adherence factor reported in previous studiers with $E$. coli.

We attempted to maximize the sensitivity of our essay by using a low colony count and defining activity as a $50 \%$ reduction in colony counts. We did this as we believe the anti-microbial activity of natural products may be more subtle than those noted in commercial antibiotics where a two log reduction in colony count may be expected.

Further studies are warranted to better understand the nature of the factors responsible for the anti-microbial activity in urines following cranberry capsules ingestion, as well as to better understand the bacterial spectrum of these factors and how they might be enhanced including a determination of optimal dosing. A study with higher concentration of CB-PVP and testing of bacterial adhesion is in progress.

\section{Conclusion}

Following ingestion of cranberry, anti-microbial activity was seen with $K$. pneumoniae. Additional studies are also 
needed to further explore the anti-microbial activity using different formulations (juice, capsule) and doses. Other studies should also explore the possibility of optimizing the anti-microbial activity using cranberry in combination with other fruits or supplements.

Competing Interests: Dr Edward Shanbrom is the inventor of Shanstar and holds the patent for it.

\section{References}

1. Blumenthal MCC, Ferrier GKL. Total sales of herbal supplements in United States show steady growth. Herbal Gram 2006;71:64-66.

2. Jepson RGML, Craig J. Cranberries for preventing urinary tract infections. Cochrane Database Syst Rev 2004;2:Art. No. CD001321.pub001323.

3. Jepson RGML, Craig J. Cranberries for treating urinary tract infections. The Cochrane Database of Systematic Reviews 1998 2004:4:Art. No. CD001322.

4. Lilja JJBJ, Neuvonen PJ. Effects of daily ingestion of cranberry juice on the pharmacokinetics of warfarin, timzanidine, and midazolam-probes of CYP2c9, VYP1A2, and CYP3A4. Clin Pharmacol Ther 2007;6:833-39.

5. Li ZSN, Carpenter CL, Thames G, Minutti C, Bowerman S. Cranberry does not affect prothrombine time in male subjects on Warfarin. J Am Diet Assoc 2006;12:2057-61.

6. Foo LYPLJ. The phytochemistry of proanthocyanidin polymers. Phytochenistry 1980;19:1747-54.

7. Jackson B, Hicks LE. Effect of cranberry juice on urinary $\mathrm{pH}$ in older adults. Home Healthc Nurse 1997;15:198-202.

8. Kinney AB, Blount M. Effect of cranberry juice on urinary $\mathrm{pH}$ Nurs Res 1979;28:287-90.
9. Zafriri D, Ofek I, Adar R, Pocino M, Sharon N. Inhibitory activity of cranberry juice on adherence of type 1 and type $\mathrm{P}$ fimbriated Escherichia coli to eucaryotic cells. Antimicrob Agents Chemother 1989;33:92-98.

10. Foo LY, Lu Y, Howell AB, Vorsa N. A-Type proanthocyanidin trimers from cranberry that inhibit adherence of uropathogenic P-fimbriated Escherichia coli. J Nat Prod 2000;63:1225-28.

11. Howell AB, Vorsa N, Der Marderosian A, Foo LY. Inhibition of the adherence of P-fimbriated Escherichia coli to uroepithelial-cell surfaces by proanthocyanidin extracts from cranberries. $N$ Engl $J$ Med 1998;339:1085-86.

12. Ahuja S, Kaack B, Roberts J. Loss of fimbrial adhesion with the addition of Vaccinum macrocarpon to the growth medium of P-fimbriated Escherichia coli. J Urol 1998;159:559-62.

13. Lee YL, Owens J, Thrupp L, Cesario TC. Does cranberry juice have antibacterial activity? JAMA 2000;283:1691.

14. Monroy-Torres R, Macias AE. Does cranberry juice have bacteriostatic activity? Rev Invest Clin 2005;57:442-46.

15. Jepson RG, Mihaljevic L, Craig J. Cranberries for preventing urinary tract infections. Cochrane Database Syst Rev 2004;2:CD001321.

16. Howell AB, Foxman B. Cranberry juice and adhesion of antibioticresistant uropathogens. JAMA 2002;287:3082-83.

17. Howell AB, Reed JD, Krueger CG, Winterbottom R, Cunningham DG, Leahy M. A-type cranberry proanthocyanidins and uropathogenic bacterial anti-adhesion activity. Phytochemistry 2005:66:2281-91.

18. Ohnishi R, Ito H, Kasajima N, Kaneda M, Kariyama R, Kumon $\mathrm{H}$, et al. Urinary excretion of anthocyanins in humans after cranberry juice ingestion. Biosci Biotechnol Biochem 2006;70:1681-87.

19. Tong H HS, Chang S. Effect of Ingesting cranberry juice on bacterial growth in urine. Am $J$ Health Syst Pharm 2006;63:1417-19.

Received March 14, 2007; accepted December 18, 2007 


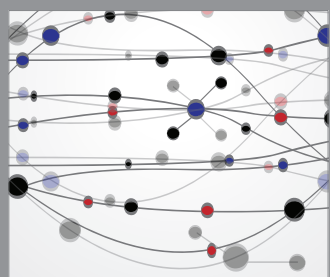

The Scientific World Journal
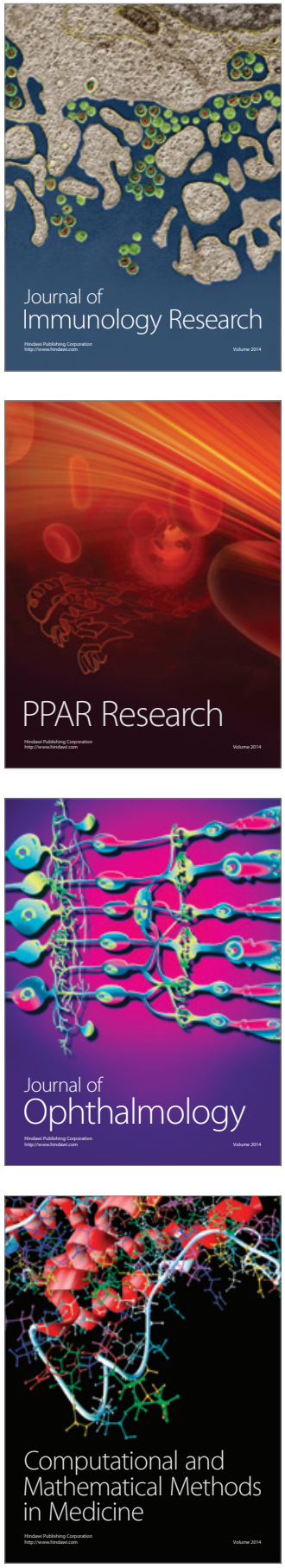

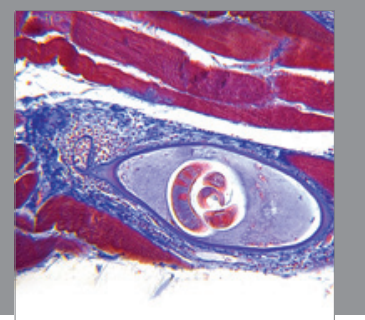

Gastroenterology

Research and Practice
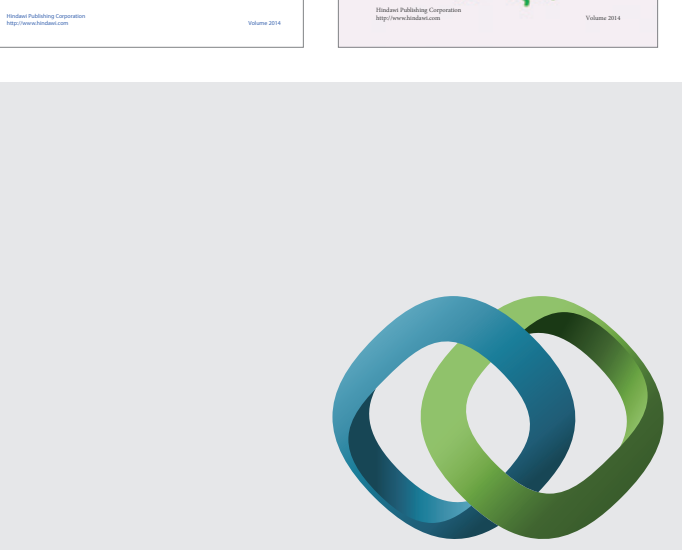

\section{Hindawi}

Submit your manuscripts at

http://www.hindawi.com
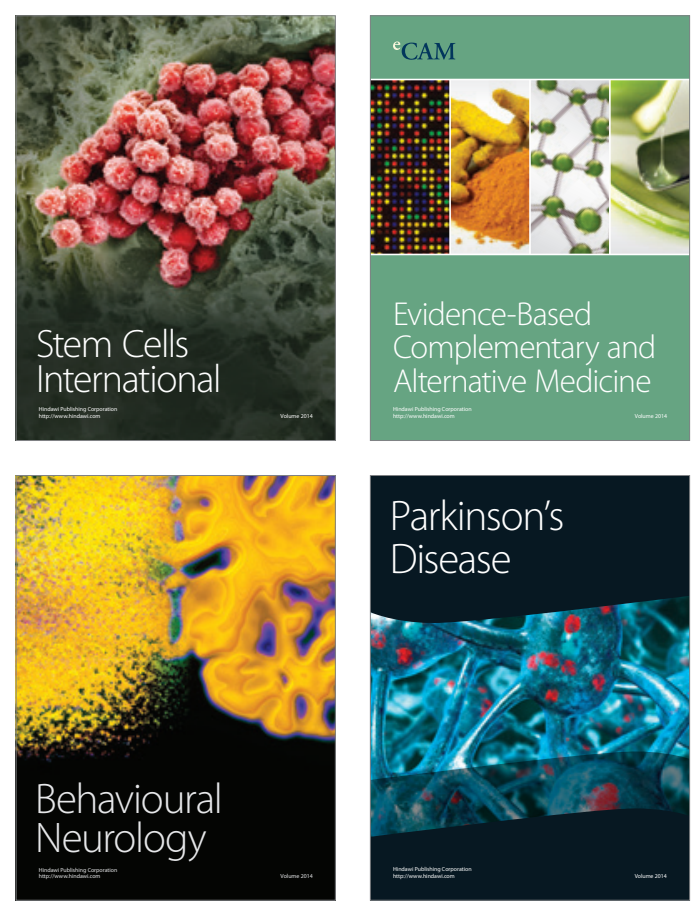

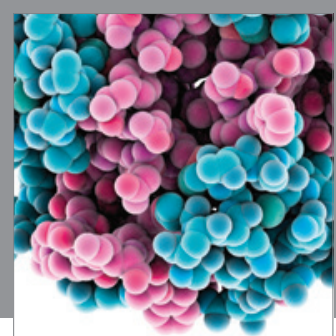

Journal of
Diabetes Research

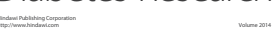

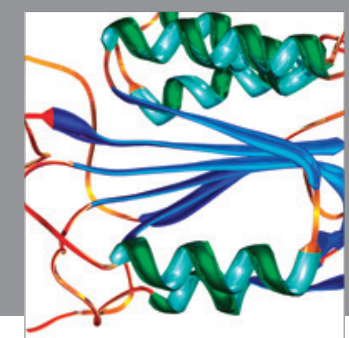

Disease Markers
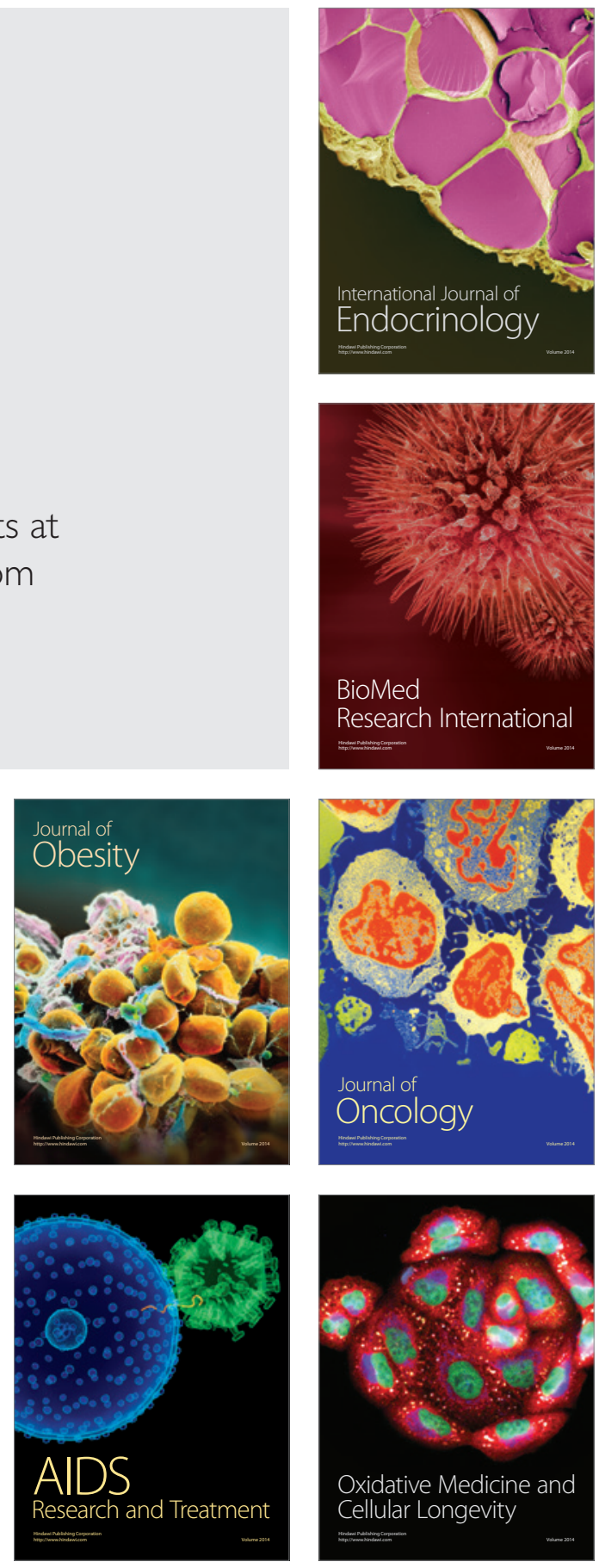